\title{
Relating paths in transition systems: the fall of the modal mu-calculus ${ }^{\star}$
}

\author{
Cătălin Dima ${ }^{1}$, Bastien Maubert ${ }^{2}$, and Sophie Pinchinat ${ }^{3}$ \\ 1 Université Paris Est, LACL, UPEC, Créteil, France. \\ dima@u-pec.fr \\ 2 LORIA - CNRS / Université de Lorraine, Nancy, France. \\ bastien.maubert@gmail.com \\ 3 Université de Rennes 1, IRISA, Rennes, France. \\ sophie.pinchinat@irisa.fr
}

\begin{abstract}
We revisit Janin and Walukiewicz's classic result on the expressive completeness of the modal mu-calculus w.r.t. MSO, when transition systems are equipped with a binary relation over paths. We obtain two natural extensions of MSO and the mu-calculus: MSO with path relation and the jumping mu-calculus. While "boundedmemory" binary relations bring about no extra expressivity to either of the two logics, "unbounded-memory" binary relations make the bisimulation-invariant fragment of MSO with path relation more expressive than the jumping mu-calculus: the existence of winning strategies in games with imperfect-information inhabits the gap.
\end{abstract}

\section{Introduction}

Monadic second-order logic (MSO) is a standard for comparing expressiveness of other logics of programs. Ground-breaking expressiveness results on MSO were obtained first on "freely-generated" structures (words, trees, tree-like structures, etc.) $[26,29]$, then on "non-free" structures like grids [18] or infinite graphs generated by regularity-preserving transformations $[10,8]$. Some attention has also been brought to the study of enrichments of MSO with unary predicate symbols or with the "equal level" binary predicate $\left(\mathrm{MSO}^{e q l}\right)[11,25]$.

Many of these expressiveness results relate MSO with automata and modal logics, among which Janin and Walukiewicz's seminal result [17] showing that the bisimulation-invariant fragment of MSO interpreted over transition systems is captured by the $\mu$-calculus. Notable exceptions to the classical trilogy between MSO, modal logics and automata are MSO on infinite partial orders (see [23] for partial results) and $\mathrm{MSO}^{e q l}$ (partial results can be found in [25]).

On the other hand, more recently there has been an increased interest in the expressiveness and decidability of logics defined on structures in which two "orthogonal" relations are considered: the so-called temporal epistemic (multiagent) logics [12], which combine time-passage relations and epistemic relations on the histories of the system $[12,15]$. A natural question that arises is whether

* Catalin Dima acknowledges financial support from the ANR project EQINOCS. Bastien Maubert acknowledges financial support from the ERC project EPS 313360. 
there exists a natural extension of MSO, of the $\mu$-calculus, and of tree automata for the temporal epistemic framework, and how would they compare?

Note that appropriate extensions of MSO, of the $\mu$-calculus and of tree automata would rely on two sorts of binary relations: those related to dynamic behaviour and those related to epistemic features. While the temporal part of these logics naturally refers to a tree-like structure, the epistemic part requires, in order to model e.g. powerful agents with "perfect recall", to consider binary relations defined on histories. Such models neither are tree-like structures, nor grid-like structures, nor graphs within the Caucal hierarchy. The only proposals in this direction that we know about are $[27,24,1]$ and [19]. [27] mentions an encoding of LTL with knowledge into Chain Logic with equal-level predicate, a fragment of $\mathrm{MSO}^{e q l}$. [24] introduces the epistemic $\mu$-calculus and studies its model-checking problem. [1] studies temporal logics over tree models with "jumpedges" which capture observational indistinguishability. Each one considers only one particular relation on histories, all three akin to synchronous perfect recall. [19] proposes a generalization of tree automata, called jumping tree automata, applying them to the study of temporal epistemic logics.

In this paper, we propose natural extensions of MSO and the $\mu$-calculus, respectively called $M S O$ with path relation $\left(\mathrm{MSO}^{\wedge}\right)$ and the jumping $\mu$-calculus $\left(\mathrm{L}_{\mu}^{\sim}\right) . \mathrm{MSO}^{\triangleleft}$ is MSO with an additional binary predicate, $\backsim$, interpreted "transversely" on the tree structure, according to the path relation. $\mathrm{L}_{\mu}^{\sim}$ is a generalization of the epistemic $\mu$-calculus defined in [24]: it features a jumping modality whose semantics relies on the path relation, generalizing the knowledge operator $K$. The path relations that we consider are arbitrary and thus cover all variants of indistinguishability relations of, e.g. [16], as well as settings not particularly related to any epistemic interpretation [13]. We investigate whether $L_{\mu}^{u}$ is expressive complete with regards to the bisimulation-invariant fragment of $\mathrm{MSO}^{\natural}$. For the class of recognizable path relations (finite memory), we observe (Theorem 1) that they add no expressivity w.r.t. the classical case hence the expressive completeness holds. We show however that the case of regular path relations breaks this completeness: the class of reachability games with imperfect information and synchronous perfect recall [5] where the first player wins cannot be defined in the jumping $\mu$-calculus, while being closed under bisimulation and definable in our extension of MSO (Theorem 2). As an intermediate result, we show that the jumping tree automata defined in [19] are equivalent to the jumping $\mu$-calculus.

The paper runs as follows: in Section 2, we develop the framework of our study on transition systems. We introduce in Section 3 the two extensions of MSO and the $\mu$-calculus, and state our results on expressive completeness (Theorem 1 and 2). In Section 4 we establish Theorem 1 and prove that jumping automata are equivalent to the jumping $\mu$-calculus, thanks to which we establish succinctness and complexity results on the jumping $\mu$-calculus with recognizable path relation. In Section 5 we establish our main result (Theorem 2) by proving that winning in reachability games with imperfect information and synchronous perfect recall is not definable in the jumping $\mu$-calculus, unless the winning con- 
ditions are observable (Theorem 3). We conclude, comment on the impacts of our results, and give some perspectives in Section 6 .

\section{Preliminary notions}

We first fix a few basic notations. Given two words $w$ and $w^{\prime}$ over some alphabet $\Sigma$, we write $w \preceq w^{\prime}$ if $w$ is a prefix of $w^{\prime}$; if $w=a_{0} a_{1} \ldots \in \Sigma^{\omega}$ is an infinite word we let, for each $i \geq 0, w[i]:=a_{i}$ and $w[0, i]:=a_{0} a_{1} \ldots a_{i}$. For a finite word $w=a_{0} \ldots a_{n-1} \in \Sigma^{*}$, its length is $|w|:=n$. Also, given a binary relation $R \subseteq A \times B$ for every $a \in A$, we let $R(a):=\{b \mid(a, b) \in R\}$. In the rest of the paper, we fix $\mathcal{A P}=\left\{p, p^{\prime}, \ldots\right\}$ a countable set of atomic propositions and $\mathcal{A} c t=\left\{a, a^{\prime}, \ldots\right\}$ a countable set of actions.

Definition 1. A transition system (over $\mathcal{A P}$ and $\mathcal{A}$ ct) is a structure $\mathcal{S}=$ $\left(S, s_{\iota},\left\{a^{\mathcal{S}}\right\}_{a \in \mathcal{A} c t},\left\{p^{\mathcal{S}}\right\}_{p \in \mathcal{A P}}\right)$, where $S$ is a countable set of states, $s_{\iota}$ is an initial state, each $a^{\mathcal{S}}$ is a binary relation over $S$ and each $p^{\mathcal{S}}$ is a subset of $S$.

The logics that we aim at are concerned with paths $^{4}$ in transition systems, that is, on their tree unfoldings. To ease the presentation, our trees have at most countable branching degree, but, unless otherwise stated, our results still hold for arbitrary degree. A tree is a nonempty, prefix-closed set $\tau \subseteq \mathbb{N}^{*}$. An element $x \in \tau$ is a node, and the empty word $\epsilon$ is the root of the tree. If $x \cdot i \in \tau$, $x \cdot i$ is a child of $x$. A node with no child is a leaf. A branch is a sequence of nodes in $\tau$ (either finite or infinite) in which each node but the first one is a child of the previous one; a branch is maximal if it is infinite or it ends up in a leaf. We write $x \preceq y$ if $y$ can be found on some branch that starts in $x$, and we let $[\tau]_{x}=\{y \mid x \preceq y\}$ denote the subtree of $\tau$ rooted in $x$. A marked tree (over $\mathcal{A P}$ and $\mathcal{A c t}$ ) is a pair $t=(\tau, m)$, where $\tau$ is a tree and $m: \tau \rightarrow\left(\mathcal{A} c t \times 2^{\mathcal{A P}}\right)$ is a marking of the nodes, where $m(x)=(a, \ell)$ means that $x$ was reached through action $a$ and $\ell$ is the set of atomic propositions that hold in $x$; we may use notation $a^{x}$ and $\ell^{x}$ for $a$ and $\ell$ when $m(x)=(a, \ell)$. Node $y$ is an $a$-child of a node $x$ if $y$ is a child of $x$ and $a^{y}=a$. The word of a node $x$ is $w(x):=m(\epsilon) m\left(x_{1}\right) \ldots m\left(x_{n}\right)$, where $\epsilon x_{1} \ldots x_{n}(=x)$ is the (unique) branch from the root to $x$. For a finite subset $A P \subset \mathcal{A P}$, an $A P$-tree is a labeled tree $t=(\tau, m)$ such that $\ell^{x} \subseteq A P$, for every node $x \in t$ (i.e. $\left.x \in \tau\right)$.

Definition 2. Let $\mathcal{S}=\left(S, s_{\iota},\left\{a^{\mathcal{S}}\right\}_{a \in \mathcal{A} c t},\left\{p^{\mathcal{S}}\right\}_{p \in \mathcal{A P}}\right)$ be a transition system. The unfolding $t_{\mathcal{S}}$ of $\mathcal{S}$ is the marked tree $(\tau, m)$ with least tree $\tau$ such that: $\epsilon$ is associated ${ }^{5}$ to $s_{\iota}$ and $\ell^{\epsilon}=\left\{p \mid s_{\iota} \in p^{\mathcal{S}}\right\}$, and for each node $x \in \tau$ associated to state $s$, if $\left\langle s \stackrel{a_{i}}{\longrightarrow} s_{i}\right\rangle_{i \in I}$ is an enumeration of the outgoing transitions from $s$ (with $I \subseteq \mathbb{N}$ ), then for each $i \in I$ we have $x \cdot i \in \tau, x \cdot i$ is associated to $s_{i}$ and $m(x \cdot i)=\left(a_{i},\left\{p \in \mathcal{A P} \mid s_{i} \in p^{\mathcal{S}}\right\}\right)$.

\footnotetext{
4 i.e. finite sequences of states and actions that start in the initial state and follow the binary relations

5 The notion of "associated state" is only used to define unfoldings and is left informal.
} 
Because in the following only actions and atomic propositions matter, the ordering of children nodes in trees is irrelevant, and the unfolding $t_{\mathcal{S}}$ is therefore uniquely defined up to isomorphism.

Definition 3. We call path relation a binary relation over $\left(\mathcal{A c t} \times 2^{\mathcal{A P}}\right)^{*}$.

A path relation links finite paths of transition systems over $\mathcal{A P}$ and $\mathcal{A} c t$. It also induces a binary relation between nodes of marked trees (over $\mathcal{A P}$ and $\mathcal{A c t}$ ) in a natural way by relating nodes $x$ and $y$ whenever their words $w(x)$ and $w(y)$ are related. We use notation $\checkmark$ for path relations.

Finally, we recall the classic notion of bisimulation [20].

Definition 4. A bisimulation between transition systems $\mathcal{S}$ and $\mathcal{S}^{\prime}$ is a binary relation $\mathcal{Z} \subseteq S \times S^{\prime}$ such that, for all $\left(s, s^{\prime}\right) \in \mathcal{Z}$, for all $p \in \mathcal{A P}$ and $a \in \mathcal{A}$ ct:

1. $s \in p^{\mathcal{S}}$ iff $s^{\prime} \in p^{\mathcal{S}^{\prime}}$;

2. for all $r \in a^{\mathcal{S}}(s)$, there is $r^{\prime} \in a^{\mathcal{S}^{\prime}}\left(s^{\prime}\right)$ such that $\left(r, r^{\prime}\right) \in \mathcal{Z}$;

3. and vice-versa.

We write $\mathcal{S} \Leftrightarrow \mathcal{S}^{\prime}$ whenever there is a bisimulation $\mathcal{Z}$ between $\mathcal{S}$ and $\mathcal{S}^{\prime}$ such that $\left(s_{\iota}, s_{\iota}^{\prime}\right) \in \mathcal{Z}$. A class $\mathcal{C}$ of transition systems is closed under bisimulation, or bisimulation closed if $\mathcal{S} \in \mathcal{C}$ and $\mathcal{S} \leftrightarrow \mathcal{S}^{\prime}$ imply $\mathcal{S}^{\prime} \in \mathcal{C}$, for all $\mathcal{S}$ and $\mathcal{S}^{\prime}$.

\section{Expressive Completeness Issues}

We fix a countable set of second order variables $\operatorname{Var}=\{X, Y, \ldots\}$. Given a marked tree $t=(\tau, m)$, a valuation is a mapping $V: \operatorname{Var} \rightarrow 2^{\tau}$. For $X \in \operatorname{Var}$ and $T \subseteq \tau$, we let $V[T / X]$ be the valuation that maps $X$ to $T$, and which coincides with $V$ on all other variables.

Monadic second order logic with path relation $\left(\mathrm{MSO}^{\wedge}\right)$ is an extension of MSO interpreted over transition systems with a path relation. Its syntax is as follows:

$$
\psi::=\operatorname{sr}(X)|p(X)| \operatorname{succ}(X, Y)|X \subseteq Y| \neg \psi\left|\psi \vee \psi^{\prime}\right| \exists X . \psi(X) \mid X \backsim Y
$$

where $p \in \mathcal{A P}$ and $X, Y \in$ Var.

An $\mathrm{MSO}^{\natural}$ formula $\psi$ is interpreted over a marked tree $t=(\tau, m)$ with a valuation $V$ and a fixed path relation $\backsim$; the fact that $t$ with valuation $V$ satisfies $\psi$ is written $t, V \models^{\star} \psi$, defined inductively as follows:

$t, V \models \operatorname{sr}(X)$ if $V(X)=\{\epsilon\}$

$t, V \models p(X)$ if for all $x \in V(X), p \in \ell^{x}$

$t, V \models \operatorname{succ}(X, Y)$ if $V(X)=\{x\}, V(Y)=\{y\}$, and $y$ is a child of $x$

$t, V \models X \subseteq Y$ if $V(X) \subseteq V(Y)$

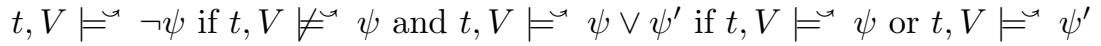

$t, V \models \exists X . \psi(X)$ if there is $T \subseteq t$ s.t. $t, V[T / X] \models{ }^{\triangleleft} \psi(X)$

$t, V \models X \backsim Y$ if $V(X)=\{x\}, V(Y)=\{y\}$, and $x \backsim y$

If $\psi \in \mathrm{MSO}^{\natural}$ has no free variable, we simply write $t \models^{\natural} \psi$, and $\mathcal{S} \models^{\natural} \psi$ whenever $t_{\mathcal{S}} \models^{\natural} \psi$, for any transition system $\mathcal{S}$. Let $\mathcal{L}(\psi, \backsim):=\left\{\mathcal{S} \mid \mathcal{S} \models^{\natural} \psi\right\}$. 
The jumping $\mu$-calculus. The syntax of the $\backsim$-jumping $\mu$-calculus $\mathrm{L}_{\mu}^{u}$ is:

$$
\varphi::=X|p| \neg \varphi|\varphi \vee \varphi| \Leftrightarrow \varphi|\Leftrightarrow \varphi| \mu X . \varphi(X)
$$

where $X \in \operatorname{Var}, p \in \mathcal{A P}, a \in \mathcal{A c t}$, and in the last rule, $X$ appears only under an even number of negations (i.e. positively) in $\varphi(X)$. We classically define the dual operators $\wedge, \square, \exists$ and $\nu$, e.g. $\square \varphi:=\neg(\hat{a} \neg \varphi$, and $\nu X . \varphi:=\neg \mu X . \neg \varphi$.

Given a path relation $\backsim$, formulas of $L_{\mu}^{u}$ are interpreted over a marked tree with a valuation $V: \operatorname{Var} \rightarrow 2^{\tau}$. We inductively define $\llbracket \varphi \rrbracket_{ज}^{t, V} \subseteq \tau$ with $t=(\tau, m)$ :

$$
\begin{aligned}
& \llbracket X \rrbracket_{\leftrightarrow}^{t, V}=V(X) \quad \llbracket p \rrbracket_{ज}^{t, V}=\left\{x \in t \mid p \in \ell^{x}\right\} \quad \llbracket \neg \varphi \rrbracket_{ज}^{t, V}=t \backslash \llbracket \varphi \rrbracket_{\leftrightarrow}^{t, V}
\end{aligned}
$$

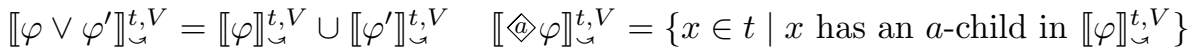

$$
\begin{aligned}
& \llbracket \Leftrightarrow \varphi \rrbracket_{\sharp}^{t, V}=\left\{x \in t \mid \text { there exists } y \in \llbracket \varphi \rrbracket_{\sharp}^{t, V} \text { such that } x \backsim y\right\} \\
& \llbracket \mu X . \varphi(X) \rrbracket_{ज}^{t, V}=\bigcap\left\{T \subseteq t \mid \llbracket \varphi(X) \rrbracket_{ज}^{t, V[T / X]} \subseteq T\right\}
\end{aligned}
$$

Note that, for each formula $\mu X . \varphi(X)$, function $T \mapsto \llbracket \varphi(X) \rrbracket_{M}^{t, V[T / X]}$ is monotone, and hence has a least fixpoint, namely $\llbracket \mu X . \varphi(X) \rrbracket_{G}^{t, V}$. If $\varphi \in \mathrm{L}_{\mu}^{u}$ has no free variables, we write $t \models^{\natural} \varphi$ whenever $\epsilon \in \llbracket \varphi \rrbracket^{t}$, and $\mathcal{S} \models^{\natural} \varphi$ whenever $t_{\mathcal{S}} \models^{\natural} \varphi$, for any transition system $\mathcal{S}$. We let $\mathcal{L}(\varphi, \leadsto):=\left\{\mathcal{S} \mid \mathcal{S} \models^{\natural} \varphi\right\}$.

Expressive Completeness. For a logic $\mathcal{L}$, a class $\mathcal{C}$ of transition systems is $\mathcal{L}$ definable if there is a formula of $\mathcal{L}$ whose set of models is exactly $\mathcal{C}$.

Proposition 1. For every path relation $\backsim$, every $L_{\mu}^{\sim}$-definable class is closed under bisimulation.

This result follows from Lemma 1 below.

Let $\backsim$ be a path relation. Note that a marked tree $t$ can be turned into a transition system $t^{\triangleleft}$ over $\mathcal{A} \mathcal{P}$ and $\mathcal{A} c t^{\prime}:=\mathcal{A} c t \cup\{\breve{a}\}$, where $\vec{a}$ is a fresh action symbol, by letting $y \in \breve{a}^{t}{ }^{\natural}(x)$ whenever $x \backsim y$. The following lemma states that if two transition systems are bisimilar (w.r.t. the transition relations only), then their unfoldings enriched with a path relation are bisimilar w.r.t. both the transition relations and the path relation.

Lemma 1. Let $\mathcal{S}$ and $\mathcal{S}^{\prime}$ be two transition systems, and let $\backsim$ be a path relation. If $\mathcal{S} \leftrightarrow \mathcal{S}^{\prime}$, then $t_{\mathcal{S}}^{\hookrightarrow} \leftrightarrow t_{\mathcal{S}^{\prime}}$.

Proposition 2. Every $L_{\mu}^{\sim}$-definable class is $\mathrm{MSO}^{\hookrightarrow}$-definable.

Proposition 2 can easily be established with a straightforward extension of the effective translation of $\mu$-calculus formulas into MSO given, e.g. in [14, Ch14].

We now engage our main concern, the expressive completeness of $\mathrm{L}_{\mu}^{u}$ with respect to $\mathrm{MSO}^{\hookrightarrow}$. As in [17], due to Proposition 1, this question can only be addressed for bisimulation-closed classes of transition systems. We thus seek properties on the path relation $\backsim$ so that $L_{\mu}^{u}$ is expressive complete with respect to $\mathrm{MSO}^{\natural}$, in the following sense: 
Definition 5 (Expressive completeness). $L_{\mu}^{u}$ is expressive complete with respect to $\mathrm{MSO}^{\wedge}$ if every bisimulation-closed class of transition systems that is $M S O^{u}$-definable is also $L_{\mu}^{u}$-definable.

Note that the results in [17] do not adapt to $\mathrm{MSO}^{\natural}$ since unfoldings of transition systems with a path relation are not tree-like structures.

We briefly recall the notions of recognizable, regular and rational relations, and we refer to [3] for details. Let $\Sigma$ be a finite alphabet. A binary relation over $\Sigma^{*}$ is rational if there is a transducer (i.e. a finite-state two-tape automaton) that accepts precisely the pairs of words of the relation. A binary relation (over $\left.\Sigma^{*}\right)$ is regular if it is accepted by a synchronous transducer ${ }^{6}$. The epistemic relation of an agent with asynchronous perfect recall [22] is rational whereas the epistemic relation of an agent with synchronous perfect recall $[4,5]$ is regular. A binary relation is recognizable if there is a finite-state word automaton over $\Sigma \cup\{\#\}$ that accepts words of the form $w \# w^{\prime}$, whenever $w$ and $w^{\prime}$ are related. The epistemic relation of a "bounded-memory" agent is recognizable. We say that a path relation $\backsim\left(\right.$ over $\left.\left(\mathcal{A c t} \times 2^{\mathcal{A P}}\right)^{*}\right)$ is rational (resp. regular, recognizable) if there are finite subsets $A \subset \mathcal{A c t}$ and $A P \subset \mathcal{A P}$ such that $\backsim$ is equal to some rational (resp. regular, recognizable) relation over $\left(A \times 2^{A P}\right)^{*}$. Finally, recall that recognizable relations are strictly contained in regular relations, and so are regular relations in rational relations.

Theorem 1. For any recognizable path relation, $L_{\mu}^{u}$ is expressive complete with respect to $\mathrm{MSO}^{\natural}$.

Theorem 2. There are regular (hence rational) binary relations for which $L_{\mu}^{u}$ is not expressive complete with respect to $\mathrm{MSO}^{\natural}$.

\section{Tree automata for the jumping $\mu$-calculus}

We prove that jumping tree automata (JTA), introduced in [19], are equivalent to the jumping $\mu$-calculus, which entails complexity and succinctness results for $L_{\mu}^{u}$ with recognizable path relation, and we prove Theorem 1 . We assume familiarity with two-player turn-based games.

For a set $X, \mathbb{B}^{+}(X)$ (with typical elements $\alpha, \beta \ldots$ ) is the set of formulas built with elements of $X$ as atomic propositions using only connectives $\vee$ and $\wedge$, and with $\top \in \mathbb{B}^{+}(X)$. Let $\operatorname{Dir}=\{\hat{a} \mid a \in \mathcal{A} c t\} \cup\{a \mid a \in \mathcal{A} c t\} \cup\{\Leftrightarrow, \Xi\}$ be the set of automaton directions.

Definition 6. $A$ jumping tree automaton (JTA) over $A P$ is a structure $\mathcal{A}=$ $\left(A P, Q, q_{\iota}, \delta, C\right)$ where $A P \subset \mathcal{A P}$ is a finite set of atomic propositions, $Q$ is a finite set of states, $q_{\iota} \in Q$ is an initial state, $\delta: Q \times 2^{A P} \rightarrow \mathbb{B}^{+}($Dir $\times Q)$ is a transition function, and $C: Q \rightarrow \mathbb{N}$ is a colouring function.

\footnotetext{
${ }^{6}$ i.e. it progresses at the same pace on each tape.
} 
JTAs resemble alternating tree automata [14, Ch. 9]. Action directions (i.e. (a) and $\square$ ) are meant to go down the input tree, whereas the new jump directions $\Leftrightarrow$ and $\Theta$ rely on an a priori given path relation. A JTA $\mathcal{A}$ with a path relation $\checkmark$ is written $(\mathcal{A}, \backsim)$. Acceptance is defined on a two-player parity game between Eve and Adam. Let $t=(\tau, m)$ be an $A P$-tree, and let $\mathcal{A}=\left(A P, Q, q_{\iota}, \delta, C\right)$. We define the parity game $\mathcal{G}_{A, u}^{t}=\left(V, v_{\iota}, E, V_{E}, V_{A}, C^{\prime}\right)$ whose set of positions is $V=\tau \times Q \times \mathbb{B}^{+}(\operatorname{Dir} \times Q)$, whose initial position is $v_{\iota}=\left(\epsilon, q_{\iota}, \delta\left(q_{\iota}, \ell^{\epsilon}\right)\right)$, and where a position $(x, q, \alpha)$ belongs to Eve iff $\alpha$ is of the form $\alpha_{1} \vee \alpha_{2}$, [^a, , $q^{\prime}$ ], or $\left[\Leftrightarrow, q^{\prime}\right]$. The possible moves in $\mathcal{G}_{\mathcal{A}, \backsim}^{t}$ are the following:

$$
\begin{array}{lr}
\left(x, q, \alpha_{1} \dagger \alpha_{2}\right) \rightarrow\left(x, q, \alpha_{i}\right) & \text { where } \dagger \in\{\vee, \wedge\} \text { and } i \in\{1,2\} \\
\left(x, q,\left[@, q^{\prime}\right]\right) \rightarrow\left(y, q^{\prime}, \delta\left(q^{\prime}, \ell^{y}\right)\right) & \text { where } @ \in\{@, a\} \text { and } y \text { is an } a \text {-child of } x \\
\left(x, q,\left[\Theta, q^{\prime}\right]\right) \rightarrow\left(y, q^{\prime}, \delta\left(q^{\prime}, \ell^{y}\right)\right) & \text { where } \Theta \in\{\Leftrightarrow, \Xi\} \text { and } x \backsim y
\end{array}
$$

Deadlock positions are winning for Eve if of the form $(x, q, \top),\left(x, q,\left[\square, q^{\prime}\right]\right)$ or $\left(x, q,\left[\boxminus, q^{\prime}\right]\right)$, and winning for Adam otherwise. The colouring function $C^{\prime}$ of $\mathcal{G}_{\mathcal{A}, \mathcal{u}}^{t}$ is inherited from the one of $\mathcal{A}$ by letting $C^{\prime}(x, q, \alpha)=C(q)$. We let $\mathcal{L}(\mathcal{A}, \backsim)=\left\{\mathcal{S} \mid\right.$ Eve has a winning strategy in $\left.\mathcal{G}_{\mathcal{A}, \hookrightarrow}^{t_{\mathcal{S}}}\right\}$.

\section{Proposition 3.}

(a) For every formula $\varphi \in L_{\mu}^{u}$, there is a JTA $\mathcal{A}_{\varphi}$ such that, for every path relation $\backsim, \mathcal{L}(\varphi, \backsim)=\mathcal{L}\left(\mathcal{A}_{\varphi}, \backsim\right)$,

(b) for every JTA $\mathcal{A}$, there is an $\mathcal{L}_{\mu}^{u}$-formula $\varphi_{\mathcal{A}}$ such that, for every path relation $\backsim, \mathcal{L}(\mathcal{A}, \backsim)=\mathcal{L}\left(\varphi_{\mathcal{A}}, \backsim\right)$.

Moreover, the translations are effective and linear.

When restricting to recognizable path relations, the folklore fact that recognizable relations are MSO-definable gives the following.

Proposition 4. $\mathrm{MSO}^{\hookrightarrow}$ with recognizable path relation is not more expressive than MSO.

Theorem 1 is obtained from Proposition 2, Proposition 4 and the expressive completeness of the $\mu$-calculus w.r.t. MSO. This collapse of the jumping $\mu$-calculus down to the $\mu$-calculus uses transformations that do not provide accurate complexity bounds regarding the jumping $\mu$-calculus. However, by Proposition 3 and the relationship between two-way alternating automata and classic tree automata [28] we get:

Proposition 5. The satisfiability problem for the jumping $\mu$-calculus with recognizable path relation over transition systems with bounded branching degree is EXPTIME-complete.

Proposition 6. For a fixed recognizable path relation, the jumping $\mu$-calculus with path relation over transition systems with bounded branching degree is at most exponentially more succinct than the $\mu$-calculus. 


\section{$5 \quad$ Games and the jumping $\mu$-calculus}

We focus on the property stating the existence of a winning strategy in twoplayer turned-based reachability games with imperfect information. This property gives us Theorem 2, for being bisimilar-invariant, expressible in $\mathrm{MSO}^{\star}$, but not expressible in $L_{\mu}^{u}$ (Theorem 4).

Two-player games with imperfect information [2, 9]. The players are Eve $^{\mathrm{i}}$ and Adam $^{\mathrm{i}}$. Eve ${ }^{\mathrm{i}}$ partially observes the positions, while Adam ${ }^{\mathrm{i}}$ has perfect information. Let $\mathcal{O} b s=\left\{o, o^{\prime}, \ldots\right\}$ be a countable set of observations. An imperfectinformation game arena is a tuple $G^{\mathrm{i}}=\left(V, v_{\iota},\left\{a^{G^{\mathrm{i}}}\right\}_{a \in \mathcal{A} c t},\left\{o^{G^{\mathrm{i}}}\right\}_{o \in \mathcal{O} b s}\right)$, where $V$ is a set of positions, $v_{\iota} \in V$ is an initial position, each $a^{G^{\mathrm{i}}}$ is a binary relation over $V$ and each $o^{G^{\mathrm{i}}}$ is a subset of $V$ such that $\left\{o^{G^{\mathrm{i}}}\right\}_{o \in \mathcal{O} b s}$ forms a partition of $V$. An action $a \in$ Act is available in $v \in V$ if $a^{G^{\mathrm{i}}}(v) \neq \emptyset$. We assume that some action is available in every position, and that two positions with the same observation share the same available actions. For $v \in V$, write $o_{v}$ the unique observation such that $v \in o_{v}$.

Players take turns, starting with $\mathrm{Eve}^{\mathrm{i}}$. In current position $v$, Eve ${ }^{\mathrm{i}}$ chooses an available action $a$ and Adam ${ }^{\mathrm{i}}$ chooses a new position $v^{\prime} \in a^{G^{\mathrm{i}}}(v)$. A play (resp. partial play) is an infinite (resp. finite) sequence $\pi=v_{0} a_{1} v_{1} a_{2} \ldots$ (resp. $\left.\rho=v_{0} a_{1} v_{1} \ldots a_{n} v_{n}\right)$ such that $v_{0}=v_{\iota}$ and, for all $i, v_{i+1} \in a_{i+1}^{G^{\mathrm{i}}}\left(v_{i}\right)$. In the synchronous perfect recall setting [5], Eve ${ }^{\mathrm{i}}$ remembers the whole sequence of observations that she receives, and her actions. The indistinguishability equivalence over partial plays is thus defined by: for $\rho=v_{0} a_{1} \ldots a_{n} v_{n}$ and $\rho^{\prime}=v_{0}^{\prime} a_{1}^{\prime} \ldots a_{n}^{\prime} v_{n}^{\prime}$, we let $\rho \sim \rho^{\prime}$ whenever, for all $1 \leq i \leq n, o_{v_{i}}=o_{v_{i}^{\prime}}$ and $a_{i}=a_{i}^{\prime}$.

Remark 1. Here, Eve remembers the sequence of actions. We point out that if she does not, then Theorem 3 below does not hold.

A (uniform) strategy for Eve is a partial function $\sigma:\left\{v_{\iota}\right\}(\mathcal{A} c t \cdot V)^{*} \rightarrow \mathcal{A c t}$ such that for two partial plays $\rho$ and $\rho^{\prime}$, if $\rho \sim \rho^{\prime}$, then $\sigma(\rho)=\sigma\left(\rho^{\prime}\right)$. We say that a play $\pi=v_{0} a_{1} v_{1} \ldots$ follows a strategy $\sigma$ if for all $i \geq 0, a_{i+1}=\sigma\left(v_{0} a_{1} v_{1} \ldots a_{i} v_{i}\right)$.

A (reachability) game with imperfect information $\mathcal{G}^{\mathrm{i}}$ is an imperfect-information arena $G^{\mathrm{i}}=\left(V, v_{\iota},\left\{a^{G^{\mathrm{i}}}\right\}_{a \in \mathcal{A} c t},\left\{o^{G^{\mathrm{i}}}\right\}_{o \in \mathcal{O} b s}\right)$ together with a reachability winning condition $F \subseteq V$. A strategy for Eve ${ }^{\mathrm{i}}$ is winning if every play that follows it visits $F$. $F$ is observable if for all positions $v$ and $v^{\prime}, o_{v}=o_{v^{\prime}}$ implies $\left(v \in F \Leftrightarrow v^{\prime} \in F\right)$.

Note that a game with imperfect information is a transition system over $\mathcal{A P}=\mathcal{O} b s \cup\{F\}$ and $\mathcal{A} c t$. Note also that the relation $\sim$ on partial plays induces a path relation, that we shall also write $\sim$.

Consider the class $\mathcal{R}(A, O)$ of reachability games with imperfect information where actions range over $A \subseteq \mathcal{A} c t$ and observations range over $O \subseteq \mathcal{O} b s$. To address logical definability, we restrict to games where $A$ and $O$ are finite sets. Also, because we address $\mu$-calculus definability, we close by bisimulation, so

that from now on a game is a transition system whose subsystem connected to the initial position is a game as defined above. 
Notice that the subclass $\mathcal{R}(A, O)$ where $\mathrm{Eve}^{\mathrm{i}}$ wins $^{7}$ is bisimulation closed since, according to [5], for any two imperfect-information reachability games $\mathcal{G}^{\mathrm{i}}$ and $\mathcal{G}^{\mathrm{i}^{\prime}}$, if $\mathcal{G}^{\mathrm{i}} \leftrightarrows \mathcal{G}^{\mathrm{i}^{\prime}}$, then Eve ${ }^{\mathrm{i}}$ wins in $\mathcal{G}^{\mathrm{i}}$ if, and only if, she wins in $\mathcal{G}^{\mathrm{i}^{\prime}}$.

Theorem 3. The subclass of $\mathcal{R}(A, O)$ of games with observable winning condition where Eve ${ }^{i}$ wins is definable in $L_{\mu}^{\sim}$, namely by $\mu X .\left(F \vee \bigvee_{a \in A} \backsim \square X\right)$.

Actually, a result similar to Theorem 3 can be established for parity conditions.

Theorem 4. The subclass of $\mathcal{R}(A, O)$ where Eve $e^{i}$ wins is not $L_{\mu}^{\sim}$-definable.

Theorem 4 entails Theorem 2 since the subclass of $\mathcal{R}(A, O)$ where Eve ${ }^{\mathrm{i}}$ wins is clearly $\mathrm{MSO}^{\sim}$-definable, closed under bisimulation, and the synchronous perfectrecall relation $\sim$ is regular ${ }^{8}$.

Proof of Theorem 4. We prove that Theorem 4 holds, already when we consider only two actions and one observation, i.e. $A=\left\{a_{0}, a_{1}\right\}$ and $O=\{o\}$.

The proof is dealt with by contradiction: Assume that there is a formula $\Phi_{\text {Win }} \in \mathrm{L}_{\mu}^{\sim}$ such that for every $\mathcal{G}^{\mathrm{i}} \in \mathcal{R}(A, O), \mathcal{G}^{\mathrm{i}} \models \sim \Phi_{\text {Win }}$ if, and only if, Eve ${ }^{\mathrm{i}}$ has a winning strategy in $\mathcal{G}^{\mathrm{i}}$. By Proposition 3 , there is a JTA $\mathcal{A}=\left(A P, Q, q_{\iota}, \delta, C\right)$ such that $\mathcal{L}\left(\Phi_{\text {Win }}, \sim\right)=\mathcal{L}(\mathcal{A}, \sim)$. Let $N:=|Q|+1$.

We describe $2^{N}$ (unfoldings of) games in $\mathcal{R}(A, O)$ where Eve ${ }^{\mathrm{i}}$ wins. For each one, we exhibit a winning strategy in the (perfect information) acceptance game of $\mathcal{A}$ on this unfolding. We then employ the "pigeon hole" principle to show that at least two of these "accepting" strategies can be combined into a new "accepting" strategy of Eve, entailing acceptance by $\mathcal{A}$ of a game in $\mathcal{R}(A, O)$ where Eve ${ }^{i}$ has no winning strategy, hence the contradiction.

The family of game unfoldings that we consider is depicted in Figure 1. They all share the same unmarked tree $\tau$, and for all $k \in\left\{1, \ldots, 2^{N}+2\right\},[\tau]_{y_{k}}$ is the full binary tree with action $a_{0}$ (resp. $a_{1}$ ) leading to the left (resp. right) child. Let $w_{k} \in\{0,1\}^{N}$ be the binary representation of $k-1$. Between the different trees $t_{i}$, the markings only differ on the leaves of $[\tau]_{y_{2} N_{+1}}$ and $[\tau]_{y_{2} N_{+2}}$ : for $1 \leq i \leq 2^{N}$, in $\left[t_{i}\right]_{y_{2^{N}+1}}$ and $\left[t_{i}\right]_{y_{2^{N}+2}}$, the only nodes in $F$ are $y_{2^{N}+1} \cdot w_{i}$ and $y_{2^{N}+2} \cdot w_{i}$. Finally, since Eve ${ }^{\mathrm{i}}$ is blind, her strategies are simply described by sequences of actions.

For each $1 \leq i \leq 2^{N}$, write $\mathcal{G}_{i}=\left(V^{i}, v_{\iota}^{i}, E^{i}, V_{E}^{i}, V_{A}^{i}, C^{i}\right)$ for $\mathcal{G}_{\mathcal{A}, \sim}^{t_{i}}$, i.e. the acceptance game of $\mathcal{A}$ on $t_{i}$ with relation $\sim$.

Clearly, for each $1 \leq i \leq 2^{N}$, Eve ${ }^{\mathrm{i}}$ wins $t_{i}$, and thus Eve wins $\mathcal{G}_{i}$. Let $\sigma_{i}$ be a winning strategy for Eve in each game $\mathcal{G}_{i}$. Let visit ${ }_{\sigma_{i}}: \tau \rightarrow 2^{Q}$ associate to each node of $\tau$ the set of states of $\mathcal{A}$ visited by strategy $\sigma_{i}$ : formally, visit $\sigma_{i}(x):=\{q \mid$ $\exists \pi \in \operatorname{Out}\left(\mathcal{G}_{i}, \sigma_{i}\right), \exists n \geq 0, \exists \alpha \in \mathcal{B}^{+}(\operatorname{Dir} \times Q)$ s.t. $\left.\pi[n]=(x, q, \alpha)\right\}$.

Since there are at most $2^{|Q|}$ different such sets of states, and we have $2^{N}$ strategies with $N=|Q|+1$, there exist $i \neq j$ s.t. $\operatorname{visit}_{\sigma_{i}}\left(y_{2^{N}+1}\right)=\operatorname{visit}_{\sigma_{j}}\left(y_{2^{N}+1}\right)$. Fix such a pair $(i, j)$, and define the game unfolding $t_{0}$, obtained from $t_{i}$ by replacing the subtree $\left[t_{i}\right]_{y_{2}+1}$ with $\left[t_{j}\right]_{y_{2}+1}$ (see Figure 1 ). Note that $t_{0}$ is

\footnotetext{
7 i.e. has a winning strategy

8 a one-state transducer that accepts it can easily be exhibited
} 

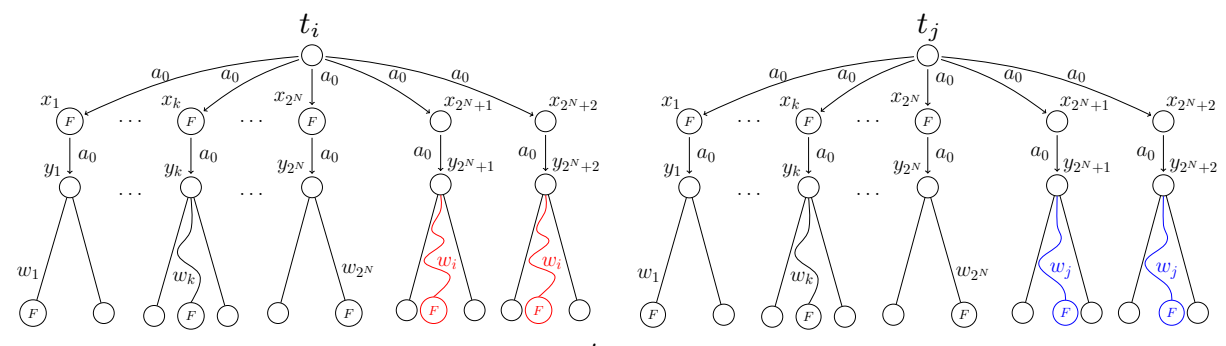

$t_{0}$

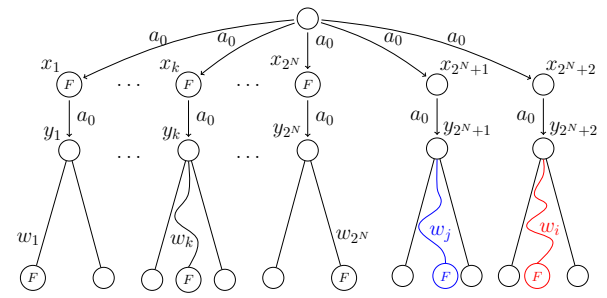

Fig. 1. The tree $t_{i}$, the tree $t_{j}$, and the hybrid tree $t_{0}$.

the unfolding of a game in $\mathcal{R}(A, O)$. Let us write $\mathcal{G}_{0}=\left(V^{0}, v_{\iota}^{0}, E^{0}, V_{E}^{0}, V_{A}^{0}, C^{0}\right)$ for $\mathcal{G}_{\mathcal{A}, \sim}^{t_{0}}$. Observe that the three games $\mathcal{G}_{i}, \mathcal{G}_{j}$ and $\mathcal{G}_{0}$ share the same set of positions: $V^{0}=V^{i}=V^{j}=\tau \times Q \times \mathbb{B}^{+}(\operatorname{Dir} \times Q)$ that we now write $V$. Also, for all $1 \leq k \leq 2^{N}+2, \ell_{0}^{y_{k}}=\ell_{i}^{y_{k}}=\ell_{j}^{y_{k}}(=\{o\})$, that we now write $\ell$. We succinctly write $v_{k}^{q}$ for positions $\left(y_{k}, q, \delta(q, \ell)\right)$, which play an important role.

The following lemma allows us to transfer the existence of winning strategies in positions $v_{k}^{q}$ from $\mathcal{G}_{i}$ and $\mathcal{G}_{j}$ to $\mathcal{G}_{0}$.

Lemma 2. 1. For all $q \in Q$ and $k \neq 2^{N}+1,\left(\mathcal{G}_{0}, v_{k}^{q}\right) \leftrightarrows\left(\mathcal{G}_{i}, v_{k}^{q}\right)$, and 2. for all $q \in Q$ and $k \neq 2^{N}+2,\left(\mathcal{G}_{0}, v_{k}^{q}\right) \leftrightarrows\left(\mathcal{G}_{j}, v_{k}^{q}\right)$.

By design of $t_{0}$, Eve ${ }^{\mathrm{i}}$ has no winning strategy in $t_{0}$. Therefore, $t_{0} \not \nvdash^{\sim} \Phi_{\mathrm{Win}}$, and thence $t_{0} \notin \mathcal{L}(\mathcal{A}, \sim)$, i.e. Eve does not have a winning strategy in the acceptance game $\mathcal{G}_{0}$ of JTA $\mathcal{A}$ on $t_{0}$ with path relation $\sim$. We establish Proposition 7 below, which provides a contradiction and terminates the proof of Theorem 4 .

Proposition 7. Eve has a winning strategy in $\mathcal{G}_{0}$.

Proof sketch. Let us define $\operatorname{Start}_{\tau}:=\left\{\epsilon, x_{1}, \ldots, x_{2^{N}+2}\right\}$, the two first levels of $\tau$, and $\operatorname{Start}_{\mathcal{G}}:=\left\{(x, q, \alpha) \in V \mid x \in \operatorname{Start}_{\tau}\right\}$. Every play in $\mathcal{G}_{0}$ starts in Start $\mathcal{G}$. Note that from any position of $\operatorname{Start}_{\mathcal{G}}$, the same moves are available in $\mathcal{G}_{0}, \mathcal{G}_{i}$ and $\mathcal{G}_{j}$. In $\mathcal{G}_{0}$, we let Eve follow $\sigma_{i}$ as long as the game is in Start $_{\mathcal{G}}$. If the game remains in Start $_{\mathcal{G}}$ for ever (by jumping infinitely), the obtained play is an outcome of $\sigma_{i}$, which is winning for Eve in $\mathcal{G}_{i}$. Because positions have the same colour in all acceptance games, this play is also winning for Eve in $\mathcal{G}_{0}$. Otherwise, the play exits Start $_{\mathcal{G}}$ by going down the tree, hence it reaches some position $v_{k}^{q}$. Because $v_{k}^{q}$ has been reached by the winning strategy $\sigma_{i}$, it is a winning position for Eve in the perfect-information parity game $\mathcal{G}_{i}$. If $k \neq 2^{N}+1$, by Point 1 of Lemma 2, i.e. $\left(\mathcal{G}_{0}, v_{k}^{q}\right) \leftrightarrows\left(\mathcal{G}_{i}, v_{k}^{q}\right)$, Eve also has a winning strategy from $v_{k}^{q}$ in $\mathcal{G}_{0}$. 
If $k=2^{N}+1$, because visit $\sigma_{i}\left(y_{2^{N}+1}\right)=\operatorname{visit}_{\sigma_{j}}\left(y_{2^{N}+1}\right), \sigma_{j}$ also visits position $v_{2^{N}+1}^{q}$, and therefore $v_{2^{N}+1}^{q}$ is a winning position for Eve in $\mathcal{G}_{j}$. By Point 2 of Lemma 2, i.e. $\mathcal{G}_{0}, v_{k}^{q} \leftrightarrow \mathcal{G}_{j}, v_{k}^{q}$, Eve also has a winning strategy from $v_{k}^{q}$ in $\mathcal{G}_{0}$.

\section{Conclusion and perspectives}

We have considered a general setting where transition systems are equipped with path relations. We have proposed natural extensions of MSO and the $\mu$-calculus in this setting: $\mathrm{MSO}^{\sim}$, which is MSO with path relations, and $\mathrm{L}_{\mu}$, which is the jumping $\mu$-calculus. We have studied the question of whether the bisimulationinvariant fragment of $\mathrm{MSO}^{\wedge}$ and $\mathrm{L}_{\mu}^{\sim}$ have the same expressivity, like in [17]. In the case of recognizable relations, the whole picture collapses to the classic case (Theorem 1). However, for the synchronous perfect recall path relation (a regular binary relation that captures models of agency with time and knowledge), the answer is negative (Theorem 2).

Our results suggest that the adequate logic on transition systems with path relations may lie in between $\mathrm{L}_{\mu}^{u}$ and $\mathrm{MSO}^{\wedge}$ : on the one hand, the latter is undecidable for regular path relations as simple as the "Equal Level" relation [25]; on the other hand fundamental decidable properties, such as winning in two-player imperfect-information games with perfect recall, are not captured by the former (Theorem 4). Remark that this property is expressible in Alternating-time Temporal Logic with imperfect information, which is therefore not subsumed by the jumping mu-calculus, but whose model-checking is decidable for one agent with synchronous perfect recall.

In addition, both the epistemic mu-calculus and imperfect-information games are decidable for perfect-recall when the knowledge of the agents is hierarchically ordered $[7,21]$. Recent results on games with imperfect information also signal other classes of models with several agents and perfect recall that can be handled [6]. So even for the case of several relations on paths, a logic that would encompass both the (jumping) mu-calculus and games with imperfect information may have good computational properties on interesting classes of systems.

\section{References}

1. R. Alur, P. Černý, and S. Chaudhuri. Model checking on trees with path equivalences. In TACAS'07, pages 664-678. Springer, 2007.

2. Krzysztof R Apt and Erich Grädel. Lectures in game theory for computer scientists. Cambridge University Press, 2011.

3. J. Berstel. Transductions and context-free languages, volume 4. Teubner Stuttgart, 1979.

4. D. Berwanger, K. Chatterjee, M. De Wulf, L. Doyen, and Thomas A. Henzinger. Strategy construction for parity games with imperfect information. Inf. Comput., 208(10):1206-1220, 2010.

5. D. Berwanger and L. Kaiser. Information tracking in games on graphs. Journal of Logic, Language and Information, 19(4):395-412, 2010.

6. Dietmar Berwanger and Anup Basil Mathew. Games with recurring certainty. In Proceedings of SR 2014, pages 91-96, 2014. 
7. R. Bozianu, C. Dima, and C. Enea. Model checking an epistemic mu-calculus with synchronous and perfect recall semantics. In TARK'2013, 2013.

8. D. Caucal. On infinite transition graphs having a decidable monadic theory. Theor. Comput. Sci., 290(1):79-115, 2003.

9. K. Chatterjee and L. Doyen. The complexity of partial-observation parity games. In LPAR 17, pages 1-14. Springer, 2010.

10. B. Courcelle and J. Engelfriet. Graph Structure and Monadic Second-Order Logic - A Language-Theoretic Approach. Cambridge University Press, 2012.

11. C. C. Elgot and M. O. Rabin. Decidability and undecidability of extensions of second (first) order theory of (generalized) successor. J. Symb. Log., 31(2):169$181,1966$.

12. R. Fagin, J. Halpern, Y. Moses, and M. Vardi. Reasoning about knowledge. The MIT Press, 2004.

13. E. Grädel. Model-checking games for logics of imperfect information. Theor. Comput. Sci., 493:2-14, 2013.

14. E. Grädel, W. Thomas, and Th. Wilke. Automata, Logics, and Infinite Games, volume 2500 of LNCS. Springer Verlag, 2002.

15. J. Y. Halpern, R. van der Meyden, and M. Y. Vardi. Complete Axiomatizations for Reasoning about Knowledge and Time. SIAM J. Comput., 33(3):674-703, 2004.

16. J. Y. Halpern and M. Y. Vardi. The complexity of reasoning about knowledge and time. 1. Lower bounds. J. Comp. Sys. Sci., 38(1):195-237, 1989.

17. D. Janin and I. Walukiewicz. On the expressive completeness of the propositional mu-calculus with respect to monadic second order logic. In Proceedings of $C O N$ CUR'96, pages 263-277. Springer, 1996.

18. O. Matz, N. Schweikardt, and W. Thomas. The monadic quantifier alternation hierarchy over grids and graphs. Inf. Comput., 179(2):356-383, 2002.

19. B. Maubert and S. Pinchinat. Jumping automata for uniform strategies. In Proceedings of FSTTCS'13, pages 287-298, 2013.

20. D. Park. Concurrency and automata on infinite sequences. In Proc. 5th GI Conf. on Th. Comp. Sci., LNCS 104, pages 167-183. Springer-Verlag, March 1981.

21. A. Pnueli and R. Rosner. On the synthesis of a reactive module. In POPL' 89 , pages 179-190. ACM Press, 1989.

22. B. Puchala. Asynchronous omega-regular games with partial information. In Proceedings of MFCS'2010, pages 592-603, 2010.

23. F. Reiter. Distributed graph automata. CoRR, abs/1404.6503, 2014.

24. N. V. Shilov and N. O. Garanina. Combining knowledge and fixpoints. Technical Report Preprint n.98, http://www.iis.nsk.su/files/preprints/098.pdf, A.P. Ershov Institute of Informatics Systems, Novosibirsk, 2002.

25. W. Thomas. Infinite trees and automaton-definable relations over omega-words. Theor. Comput. Sci., 103(1):143-159, 1992.

26. W. Thomas. Languages, automata, and logic. In G. Rozenberg and A. Salomaa, editors, Handbook of Formal Languages, volume 3, Beyond Words, pages 389-455. Springer Verlag, 1997.

27. R. van der Meyden and N. Shilov. Model checking knowledge and time in systems with perfect recall (extended abstract). In Proceedings of FSTTCS'99, volume 1738 of $L N C S$, pages 432-445, 1999.

28. M. Y. Vardi. Reasoning about the past with two-way automata. In Proceedings of ICALP'98, volume 1443, pages 628-641, 1998.

29. I. Walukiewicz. Monadic second-order logic on tree-like structures. Theor. Comput. Sci., 275(1-2):311-346, 2002. 